AUSTRALIEN

\title{
Hilfe umfassend organisieren
}

\section{Wolfgang Müller}

In Australien organisiert der Staat mit einer zentralen Anlaufstelle alle staatlichen Leistungen für seine Bürger. Dabei bezieht die "Multi-Service-Agentur Centrelink " die zivilgesellschaftlichen Ressourcen und die Wirtschaftsunternehmen mit ein.

$\mathrm{E}$ ine der größten Herausforderungen für alle öffentliche Verwaltungen im Sozialbereich dürfte es sein, einen Klienten nicht nur in seinem Status quo zu administrieren, sondern ihn durch die zur Verfügung stehenden Maßnahmen und Produkte so weit zu helfen, dass er sich selber wieder vollständig in die Gesellschaft reintegrieren kann. Bei der Betrachtung des Zielerreichungsgrades eines solchen - zugegebenermaßen ambitionierten - Ansatzes darf vermerkt werden, dass es noch erheblichen Optimierungsbedarf gibt. Wenn man den Horizont auf der Suche nach innovativen Lösungsansätzen über den europäischen Raum hinaus erweitert, so ist seit einiger Zeit ein besonders erfolgsversprechender Ansatz in Australien zu beobachten.
In Australien ist es 1997 zur Aufstellung einer Multi-Service-Agentur gekommen, deren Aufgabe es ist, öffentliche Leistungen gegenüber den Bürgern effizient und effektiv zu erbringen. Die Absicht der Regierung zielt also einerseits auf die Realisierung von Effizienzgewinnen, indem die Produktivität gesteigert wird; andererseits richtet sich das Augenmerk gleichberechtigt auf die Verbesserung der Effektivität, indem möglichst bedürfnisgerechte und umfassende Lösungsmöglichkeiten für den Leistungsempfänger gefunden werden. Gerade der letzte Anspruch spiegelt sich in so genannten »Customer Service Charters « wieder, in denen die eigenen Verpflichtungen gegenüber den Bürgern in schriftlicher Form kenntlich gemacht werden; der Anspruch auf Erfüllung kann daher mit einer entsprechenden politischen Relevanz öffentlich wirksam eingefordert werden.

Centrelink selber lässt sich am besten skizzenhaft über einige prägnante Leistungsdaten beschreiben (vgl. Tabelle S. 8). Um dem Anspruch einer im Vergleich zu den vorherigen Strukturen verbesserten Effektivität gerecht zu werden, greift Centrelink auf zwei Kon-

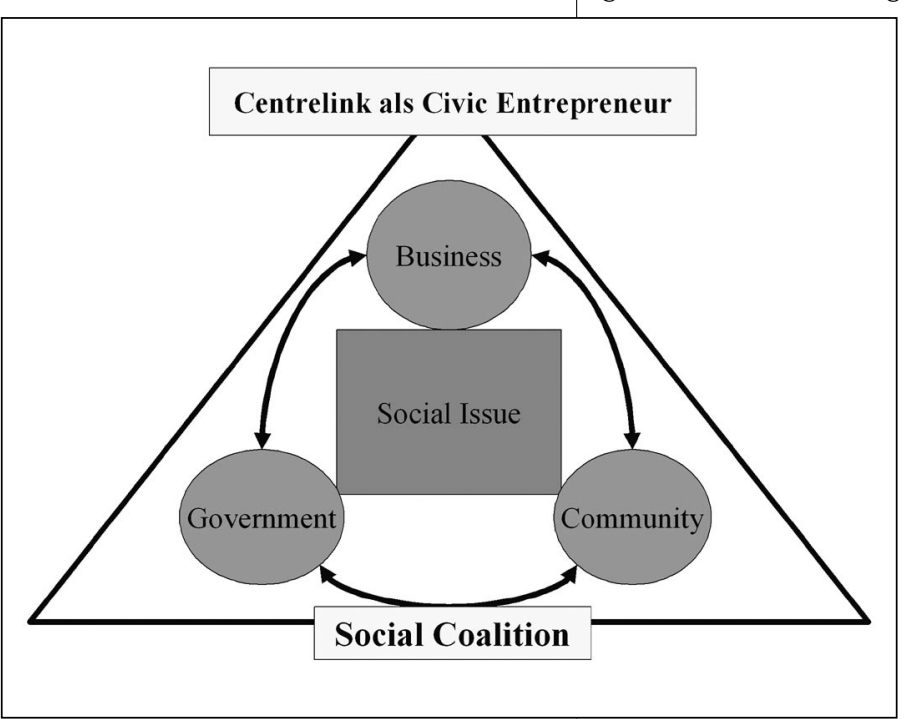
zepte zurück:

- Zunächst kommt das Konzept des »One-StopShop « zur Anwendung: Der Leistungsempfänger hat mit Blick auf staatliche Leistungen nur noch eine Verwaltungsorganisation - eben Centrelink - anzulaufen, um

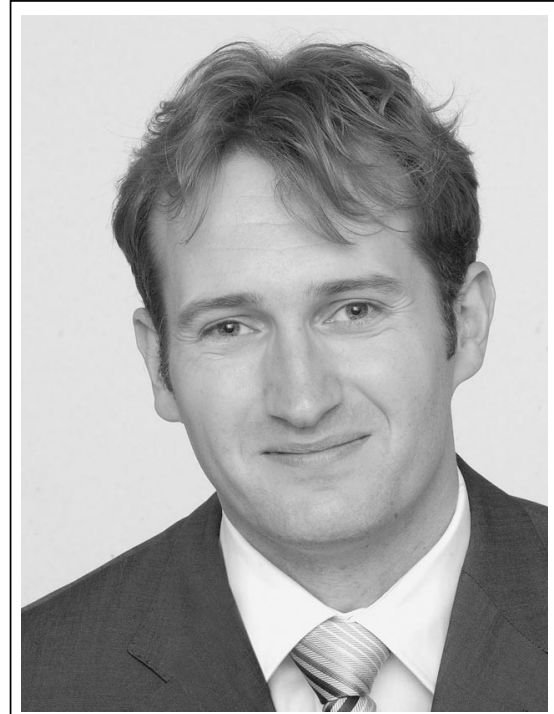

Dr. Wolfgang Müller ist wissenschaftlicher Mitarbeiter an der Helmut-Schmidt-Universität, Universität der Bundeswehr Hamburg sowie Mitglied im Arbeitskreis "Verwaltungshandeln durch flexibles Organisationsmanagement « der Arbeitsgemeinschaft für wirtschaftliche Verwaltung e. V. (AWV). Er hat über die australische Multi-Service-Agentur Centrelink promoviert; die Arbeit ist im Internet abrufbar (http://www.hsu-bibliothek.de/emedien/index.html); eine Buchpublikation ist demnächst verfügbar. E-Mailwolfgang.mueller@ unibw-hamburg.de

alle ihm potenziell zustehende Leistungen zu beantragen. Der Zugang des Bürgers zur öffentlichen Verwaltung findet dabei über Lebenslagen statt. Die Logik hinter den Lebenslagen als Zugangsmuster liegt darin, dass jeder Bürger in seinem Leben zwangsläufig mit bestimmten Situationen konfrontiert wird (beispielsweise Eheschließung, Geburt eines Kindes, Behinderung, Eintritt in die Rente etc.), denen prinzipiell alle öffentlichen (Sozial-) Leistungen zugeordnet werden können. Von daher sollte der Bürger den Kontakt zur Verwaltung nicht in Abhängigkeit der programmatischen Verantwortung einer bestimmten Leistung aufnehmen müssen, sondern vielmehr auf Grundlage seiner individuellen Lebenslage. 


\section{Beispiel Überschuldung: so arbeitet Centrelink}

Für einen der größten Problembereiche - die Überschuldung greift die Multi-Service-Agentur Centrelink in Australien mit Hilfe des sogenannten »Centrepay« auf privatwirtschaftliche Unternehmen zurück. Dabei wird ein Anteil an den wohlfahrtsstaatlichen Zahlungen, auf die ein Empfänger Anspruch hat, für bestimmte Leistungen (z. B. Miete, Strom, Wasser und Gas) vor der Auszahlung an den Empfänger von der Gesamtsumme abgezogen. Der Leistungsempfänger tätigt somit schon seine essenziell notwendigen Ausgaben, bevor er einen - dann um diese Ausgaben reduzierten Geldbetrag ausgezahlt bekommt.

Die Gefahr einer Überschuldung für die Leistungsempfänger wird reduziert, da ihre absolut notwendigen Auslagen bereits bezahlt worden sind und sie von Anfang an weniger konsumtives Geld zur Verfügung haben. Die Vorabzüge beziehen sich nur auf Leistungen, die sowieso hätten bezahlt werden müssen. Die daran beteiligten zirka 2.900 Unternehmen und Organisationen profitieren von diesem System, da sie pünktlicher ihre Zahlungen von den Leistungsempfängern erhalten, sie ausbleibenden Zahlungen nicht mehr hinterherlaufen müssen und eine Überschuldung - und damit das gänzliche Ausbleiben von Zahlungen - reduziert wird.

- Allerdings ist es offensichtlich, dass staatliche Leistungen allein im Prinzip nur eine suboptimale Lösung darstellen können, weil die gesellschaftliche Kapazität zur Lösung sozialer Notlagen ausgeklammert wird. Alleine der singuläre Geldtransfer ohne auch nur den Versuch der Lösung assoziierter Probleme ist oft nicht ausreichend, die soziale Notlage wirklich zu beheben. Das Konzept des »OneStop-Shop« wird daher durch eine zweites Konzept - den »First-StopShop« - ergänzt. In diesem Konzept tritt Centrelink in die Rolle eines so genannten »Civic Entrepreneur «, der als Schaltstelle eine "Social Coalition « zwischen Staat, Wirtschaft und gemeinnützigen Organisationen unterhält (vgl. Grafik S. 6).

Grundannahme dieses Konzeptes ist zum einen, dass der Bürger auf jeden Fall die öffentliche Verwaltung für den Erhalt wohlfahrtsstaatlicher Leistungen kontaktiert; zum anderen ist es offensichtlich, dass gerade gemeinnützige Organisationen oft nicht die personellen und finanziellen Ressourcen haben, eine regionale - geschweige denn eine landesweite Kontaktstruktur für ihre potenziellen Leistungsabnehmer aufzubauen. Da die öffentliche Verwaltung - in diesem Fall Centrelink - jedoch bereits eine ausdifferenzierte und flächendeckende Struktur vorhält, kann man diese doch für einen ganzheitlichen Problemlösungsansatz unter Einbeziehung gemeinnütziger Organisationen nutzen.

Geht man nun von der konzeptionellen zur instrumentellen Ebene über, so finden sich schon auf der Ebene der Sachbearbeitung erste Elemente eines gemeinsamen Ansatzes. Der alleine auf die Bereitstellung öffentlicher Leistungen spezialisierte Sachbearbeiter wird funktional durch kundengruppenspezifische Mitarbeiter ergänzt.

Mit Blick auf die beiden wichtigsten Kundengruppen von Centrelink sind dies der »Disability Officer « für die behinderten Menschen (rund 1,1 Millionen oder 18,5 Prozent der Kunden) sowie der »Multicultural Service Officer « für Immigranten aus »NonEnglish speaking countries « (rund 1 Millionen oder 18 Prozent der Kunden). Beide werden in den Kundenzentren eingesetzt, in denen ihre jeweilige Kundengruppe anteilsmäßig besonders stark vertreten ist (also beispielsweise in Gebieten mit einen Immigrantenanteil von 55 bis 70 Prozent). Ihre Aufgaben bestehen nicht in der Vermittlung staatlicher Leistungen, auf welche die Kunden der jeweilige Gruppe Anspruch hätten. Vielmehr besteht ihre Aufgabe darin, Hilfestellungen zu geben und Kontakte herzustellen, die außerhalb der Grenzen der öffentlichen Verwaltung vorzufinden sind.

Von daher geht es zunächst um die Bildung und Aufrechterhaltung eines Netzwerkes - wie im Beispiel der
Gruppe der behinderten Menschen — zwischen entsprechenden »community organisations « (wie z. B. »Blind Citizens Australia «), privaten Unternehmen (wie z. B. »Comcare $«$ ) und anderen Verwaltungsorganisationen (wie z. B. der »Commonwealth Rehabilitation Service Australia «). Für diesen Zweck unterhält Centrelink eine dezentral einsehbare und einzuspeisende Datenbank (die so genannte »Community Connections « mit nationalen und lokalen »community information «) sowie einen eigenen Publikationsbereich auf der Centrelink-Website (Community Groups Publications).

Darüber hinaus wird die Institutionalisierung des Informations- und Erfahrungstransfers (Best Practices) zwischen staatlich-administrativen und gemeinnützigen Organisationen vorangetrieben. Der Austausch ist in beiden Fällen bis hin zur nationalen Ebene - mit der »Disability Customer Service Reference Group « sowie der »National Multicultural Reference Group « - organisiert. Dieser Transfer existiert durchaus in beide Richtungen, denn durch den engen persönlichen Kontakt zwischen den Mitarbeitern von Centrelink und den jeweiligen gemeinnützigen Organisationen ist sowohl Centrelink über die potenziellen Hilfsmöglichkeiten der gemeinnützigen Organisationen vertraut, als auch umgekehrt die gemeinnützigen Organisationen über den vollen Umfang des Leistungsspektrums der öffentlichen Verwaltung informiert sind.

In dieses Geflecht zwischen erstem und dritten Sektor werden zusätzlich noch sowohl weitere Elemente von Centrelink als auch privatwirtschaftliche Unternehmen eingebunden, um die häufig anzutreffenden sekundären Begleiterscheinungen sozialer Notlagen (Gewalt, Überschuldung, psychologische Probleme, etc.) in einem Gesamtpaket anzugehen.

Centrelink begleitet daher seine Sachbearbeitung durch proaktive und präventive soziale Elemente. Der »Social Worker « ist dabei für weitere soziale Hilfestellungen wie beispielsweise dem Finden einer adäquaten Wohnung oder einer ausreichenden wie auch gesundheitsförderlichen Ernährung zuständig, während der »Occupational Psychologist « bei Gewalt, Alkohol, Depressionen etc. eingesetzt wird. 


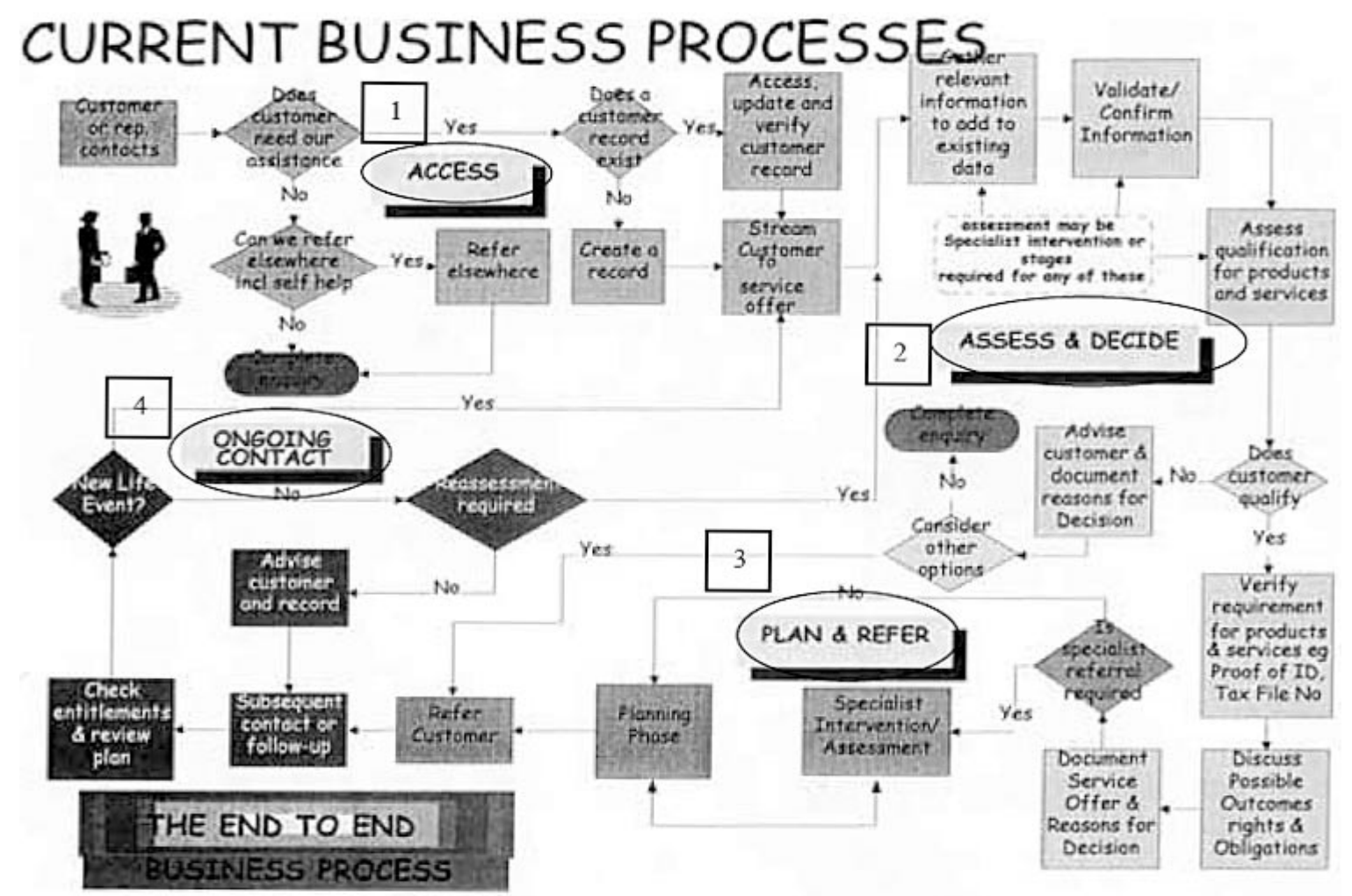

\section{Fallmanagement in Australien}

Bei Centrelink sind im Case Management alle Prozesse vier Bereichen zugeteilt worden. Die vier Bereiche sind mit »Access, Assess \& Decide, Plan \& Refer and Ongoing Contact« bezeichnet und stellen das in Anwendung befindliche Sachbearbeitungskonzept von Centrelink bei allen Lebenslagen dar. Während früher die 70 unterschiedlichen Prozesse teilweise auch noch von verschiedenen Stellen oder Personen bearbeitet worden sind, liegt die Bearbeitung der vier Teilsegmente heute nur noch in einer Hand: in der des »Customer Service Officer « als »Case-Manager «. Diese Art des Case Management kommt in den Kanälen zur Anwendung, in denen eine komplette Sachbearbeitung möglich ist (zur Zeit sind dies die »Customer Service Centre « und die »Call Centre«). Die Durchführung und Verantwortung der Vorgangsbearbeitung durch bzw. bei einem einzigen Mitarbeiter ist gewählt worden, weil selbst bei einer Unterteilung der Ablaufprozesse in vier Bereiche immer noch von einem sachlichen Zusammenhang der einzelnen Handlungen innerhalb der vier Bereiche untereinander auszugehen ist. Das australische Originaldokument zeigt die Prozessstruktur des Fallmanagements bei Centrelink.

\section{Fazit}

Das innovative Potenzial von Centrelink liegt offensichtlich darin, dass der Staat seine personellen und strukturellen Ressourcen auch gemeinnützigen Organisationen zur Verfügung stellt, um einen ganzheitlichen Problemlösungsansatz zum Wohl des (sozialen) Kunden zu realisieren. Darüber hinaus ist bei diesem Konzept zu erkennen, dass ein Ausgleich im Bereich der gegenseitigen Kenntnisse vorgenommen worden ist. Während gemeinnützige Organisationen schon immer zum Wohle ihrer Klientel über das staatliche Leistungsspektrum informiert gewesen waren, scheint es dem Staat erst durch Centrelink gelungen zu sein, das enorme Wissens- und Hilfspotenzial des dritten Sektors zu aktivieren.

\section{Leistungsdaten von Centrelink im Überblick 2004}

Anzahl der Kunden:

6.5 Millionen (bei einer Gesamteinwohnerzahl Australiens von 19 Millionen sind ca. 33 Prozent Kunden von Centrelink; bezogen auf Familien sind es sogar 85 Prozent)

Beschäftigte

Customer Service Centres 25.448

321

Call Centres

26

Access Points

Service Delivery Points

Auftraggebende Ministerien

Ausgezahltes Leistungsvolumen

Getätigte Onlinetransaktionen

159

Über 1.000 landesweit

25

Über 35 Milliarden Euro

Ca. 4.4 Milliarden

Erteilte Leistungsanspruchsberechtigungen 9.7 Millionen

Versendete Briefsendungen

Getätigte Hausbesuche

Erfolgreich getätigte Telefongespräche

Gezählte Zugriffe auf die Homepage

90.6 Millionen

9.208

28.0 Millionen

32.6 Millionen

Tabelle: Kennziffern der Multi-Service-Agentur Centrelink in Australien 